\title{
KINETIC MODELS OF EPITAXIAL GROWTH: THEORY AND EXPERIMENT*
}

\author{
D.D. VVEDENSKY and P. ŠmilaueR ${ }^{\dagger}$
}

The Blackett Laboratory, Imperial College, London SW7 2BZ, United Kingdom

We examine the morphological evolution of growing surfaces using Monte Carlo simulations of a solid-on-solid model. We use direct comparisons with experiment both to identify the kinetic processes that must be included in a model for $\mathrm{GaAs}(001)$ homoepitaxy and to parametrize the rates of these processes. We first examine the evolution of a vicinal surface during the first few monolayers of growth and compare the density of surface steps of the simulated surfaces with reflection high-energy electron-diffraction measurements. By including both a non-thermal incorporation step of freshly deposited atoms and a barrier to interlayer atomic transport, excellent quantitative agreement is obtained for an entire range of growth conditions, including the relaxation of the surface toward equilibrium upon the termination of the beam. We then examine the morphology as successively more layers are grown and find that the surface evolves into a self-organized state wherein the local slope of the growing features remains approximately constant with time.

PACS numbers: $61.50 . \mathrm{Cj}$

\section{Introduction}

Epitaxial growth is a non-equilibrium process whereby material is deposited onto a solid surface which then crystallizes in a manner determined by the crystal structure of the surface. In the simplest realization of this method, molecular-beam epitaxy (MBE), neutral atomic and molecular beams are directed towards a heated substrate in an ultra-high vacuum environment [1-3]. Neither collisions nor chemical reactions occur between the constituents of the beam prior to arrival at the substrate. Thus, the deposition is ballistic and, under typical growth conditions, the particles adsorb onto the target surface irreversibly. These facts, combined

\footnotetext{
*This work is supported by Imperial College and the Research Development Corporation of Japan under the auspices of the "Atomic Arrangement: Design and Control for New Materials" Joint Research Program.

${ }^{\dagger}$ Also at: Interdisciplinary Research Centre for Semiconductor Materials, Imperial College, London SW7 2BZ, United Kingdom; on leave from: Institute of Physics, Czech Academy of Science, Cukrovarnická 10, 16200 Praha 6, Czech Republic.
} 
with a relatively slow growth rate (typically one atomic layer per second), are responsible for the essentially monolayer control of the chemical composition during MBE.

A schematic diagram of an MBE growth chamber is shown in Fig. 1. The substrate is mounted onto a rotating substrate holder. The collimated molecular beams are generated within Knudsen cells which, for the growth of GaAs, are produced in the form of atomic $\mathrm{Ga}$ and either $\mathrm{As}_{2}$ or $\mathrm{As}_{4}$. The growth process is

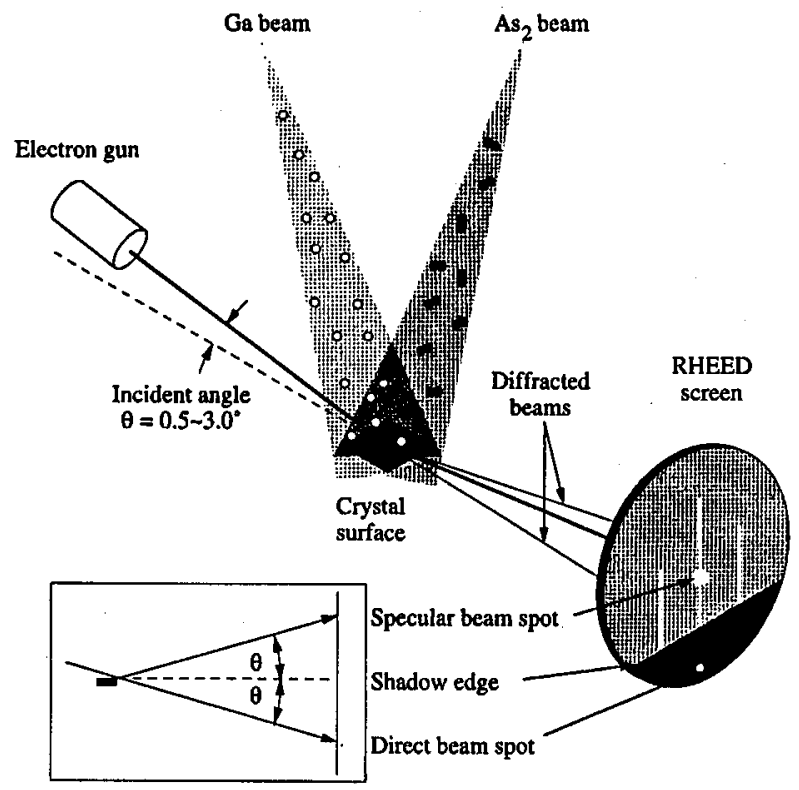

Fig. 1. Schematic illustration of a growth chamber used in MBE, showing the substrate, the Knudsen cells, and the RHEED measurement apparatus (after Ref. [4]).

monitored by a surface diffraction technique known as reflection high-energy electron diffraction (RHEED) [5], wherein a high-energy $(\approx 10 \mathrm{keV})$ electron beam is directed at a glancing angle $\left(\approx 0.5-3^{\circ}\right)$ to the substrate. The diffraction pattern can then be monitored during growth, which provides information concerning the surface reconstruction and other chemical and morphological features of the surface. The simplicity of this measurement, combined with the geometric compatibility between the electron gun, the screen and the Knudsen cells, has made RHEED a standard way of performing atomic-level "quality control" during epitaxial growth.

However, despite the widespread use of RIIEED, a direct interpretation of the diffraction pattern is not yet possible because of the strong interaction of the electrons with the surface. Thus, in contrast to atomic or X-ray scattering, for which the kinematic approximation of the diffraction pattern often suffices, a quantitative interpretation of the RHEED pattern requires a multiple scattering treatment. As a result, most of the detailed atomic level information for growing 
surface has been obtained with the scanning tunneling microscope [6]. The most common procedure for scanning as-grown surfaces is to interrupt the growth and then quench the surface to insure that the morphology of the scanned surface is as close as possible to that of the surface upon cessation of growth. An exciting variation of this procedure is to use relatively slow growth rates to enable the surface to be scanned during growth, thereby generating a motion picture of the growth process [7].

In this paper, we use simulations of a solid-on-solid model [8-10] to examine the evolution of epitaxially growing $\mathrm{GaAs}(001)$ surfaces. We will first compare simulated surface morphologies with RHEED measurements on vicinal $\mathrm{GaAs}(001)$ by using the density of surface steps as a measure of the loss of specular intensity due to surface disorder. The fact that quantitative agreement can be obtained between the simulations and the measurements over a range of growth conditions provides a level of confidence that the dominant kinetic processes have been included in the model. One such process is the effect of barriers at step-edges on interlayer atomic transport. We examine the influence of this barrier on the evolution of the growth front morphology in the multilayer regime on nominally singular $\mathrm{GaAs}(001)$ and compare with recent atomic force microscopy studies [11].

\section{The solid-on-solid model}

Much of the theoretical work on crystal growth has been based on lattice models $[8-10,12]$. In this approach, atomic sites are fixed positions on a lattice and the kinetic processes that cause transitions among these sites are described by Arrhenius-type rates

$$
K(T)=K_{0} \exp (-E / k T),
$$

where $K_{0}$ is the attempt rate, $E$ is the activation barrier, $k$ is Boltzmann's constant and $T$ is the temperature. Although being technically merely a mean field approximation to the actual microscopic process, various corrections to (1) are well understood $[13,14]$ and do not alter the essential point that the rates are determined by the transition state energy barrier $E$ that is not necessarily associated with either the initial state or the final state.

In our model of epitaxial growth $[12,15]$, the substrate is assumed to have a simple cubic structure with neither vacancies nor overhangs. Thus, every atom has an atom beneath it - the so-called solid-on-solid criterion - so every configuration of the surface is specified completely by the collection of column height variables at each site. Although the simple cubic structure and the absence of vacancies and overhangs simplifies the computational and mathematical formulations of this model, neither is an essential assumption and both can be lifted without undue difficulty [16-18].

Growth is initiated by adding atoms randomly to the columns at each site at an average rate of $\tau^{-1}$, where $\tau$ is the layer completion time. The migration of surface adatoms is taken as a nearest-neighbor hopping process whose rate is

$$
k(T)=k_{0} \exp \left(-E_{\mathrm{D}} / k T\right)
$$

where $k_{0}$ corresponds to an adatom vibrational frequency and $E_{D}$ is the hopping barrier. The temperature is assumed to be constant across the substrate [19]. 
The prefactor $k_{0}$ is taken either as $k_{0}=2 k T / h$, where $h$ is Planck's constant, or assigned a constant value $\left(\approx 10^{13} \mathrm{~s}^{-1}\right)$. The simplest form of hopping barrier is comprised of a term, $E_{\mathrm{S}}$, from the substrate, and a contribution, $E_{\mathrm{N}}$, from each lateral nearest neighbor. Thus, for an $n$-fold coordinated atom $(n=0,1,2,3,4)$, $E_{\mathrm{D}}=E_{\mathrm{S}}+n E_{\mathrm{N}}$. This barrier is assumed to depend only on the initial environment of the migrating atom and only the topmost atoms in the columns are active. To maintain the solid-on-solid constraint, we stipulate that an atom hopping at the rate given in (2) lands (with equal probability) on top of one of the four nearest-neighbor columns. Our model thus exhibits height diffusion rather than true surface diffusion $[17,18]$.

To reproduce the potential profile expected in the vicinity of a step-edge (Fig. 2), we introduce an activation barrier to hopping at step edges, $E_{\mathrm{B}}$, of the same form as that used in our simulations of the re-entrant oscillations seen in thermal-energy atom scattering measurements on $\operatorname{Pt}(111)$ [20]. This barrier is

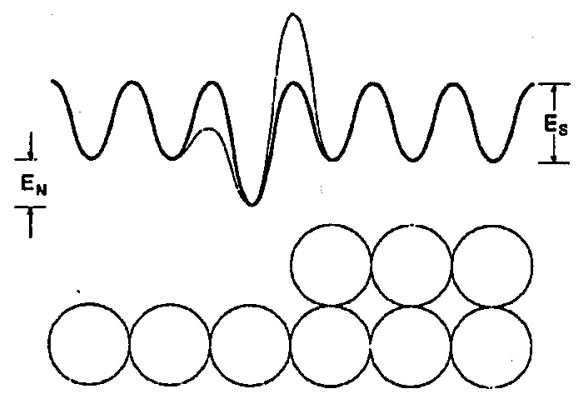

Fig. 2. Potential energy surface near a step edge. The bold curve represents the potential in a model that includes hopping with no influence of the step-edge barrier. The lighter curve is a more realistic model potential near a step edge.

expected to provide a strong signature in the recovery profile by inhibiting the interlayer transport of adatoms and thus slowing down the recovery [21]. However, even a quite small barrier to interlayer hopping has important consequences during growth as well. The surface quickly roughens and complete disagreement between simulations and experiment during growth and recovery is observed. We are thus led to the conclusion that if step-edge barriers are present then some compensating smoothing mechanism must be present as well. We have therefore included an additional process that an arriving atom undergoes before being incorporated on the surface. A search is performed within a square of a fixed linear size $L$, centered at the original site, for the site that maximizes the number of nearest neighbors. This process smooths the edges of pre-existing steps and islands created on the terraces. A similar mechanism has been invoked to explain the monolayer to bilayer transition in the growth of group IV materials [22, 23], and barriers are known to exist on metal surfaces [24-26]. However, we can only speculate about its possible microscopic origins for the case of $\mathrm{GaAs}(001)$. 


\section{Comparison with electron diffraction measurements}

In attempting to develop a model of $\mathrm{GaAs}(001)$ homoepitaxy with as few free parameters as possible while retaining the essential features of the growth kinetics, some simplifying assumptions have been introduced. Those that require the closest examination are as follows. (i) The mobility of adatoms is isotropic. (ii) The As kinetics is not included explicitly in the model, i.e., the As surface concentration is assumed to be sufficient to insure microscopic stoichiometry [27]. (iii) The effects of the surface reconstruction on adatom mobility can be incorporated into effective migration parameters $\left(E_{\mathrm{S}}, E_{\mathrm{N}}\right)$, as for $\mathrm{Si}(001)$ homoepitaxy [28].

In the experiments of Shitara et al. [29], growth and diffraction conditions were chosen to conform as closely as possible to these assumptions. In particular, to satisfy (i) the surfaces were misoriented toward the [010] direction to reduce the effect of the anisotropy. To satisfy (ii) the As/Ga (atomic) ratio was held at approximately 2.5 to maintain the $2 \times 4$ reconstruction over the temperature range of interest. This also addresses assumption (iii). The diffraction conditions used were "in-phase" or "Bragg" conditions for which the maxima in the specular-beam intensities on the corresponding singular surface occur at monolayer (ML) increments of material deposited. Since for these diffraction conditions the kinematic approximation yields a constant intensity, regardless of the surface configuration, the density of surface steps was used to model the variations of the RHEED specular-beam intensity.

The model used in Ref. [29] included only random deposition and nearest-neighbor hopping. The most striking result to emerge from this study is the quantitative agreement between the RHEED specular-beam intensity and the step density during growth to the extent that the relative changes of the two quantities with temperature are nearly the same. But there was also clear disagreement during post-growth equilibration of the surface, which was most evident at lower substrate temperatures. The simulations generally showed a much more rapid recovery than the measured RHEED profiles. More important, these simulations were unable to reproduce the systematic dependencies of the time constant for the initial stage of the recovery [30].

Motivated by these discrepancies, the corresponding comparisons were made with the model that included the non-thermal incorporation of freshly-deposited atoms and the step-edge barrier $E_{\mathrm{B}}$ to interlayer transport [31]. Representative comparisons are shown in Fig. 3. The step densities show an improved level of agreement with the RHEED curves over the recovery and growth periods compared with those described above. In particular, a better account of the first maximum delay phenomenon $[32,33]$ is obtained at the onset of growth. This is due to more atoms being incorporated into pre-existing steps immediately upon deposition, leaving fewer atoms available for cluster formation on the terraces.

The comparisons in Fig. 3 are compelling not least because the effects of the incorporation process and step-edge barrier act in opposition during growth but in concert during recovery. The step-edge barrier slows down the equilibration process by inhibiting interlayer transport while the incorporation process produces a smoothing of step edges and a decrease in the number of free adatoms, which 

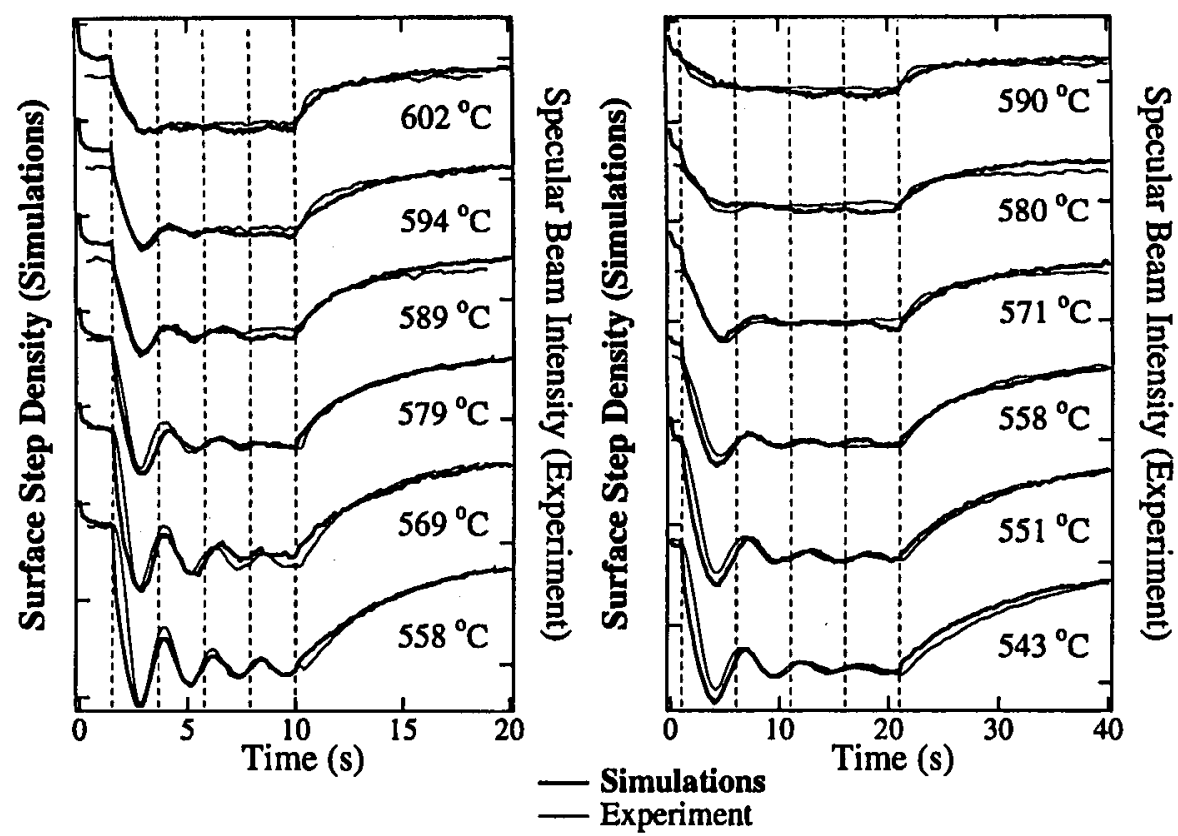

Fig. 3. Comparison of measured RHEED specular intensities and step densities from simulated surfaces during growth on vicinal $\mathrm{GaAs}(001)$ with a misorientation of $2^{\circ}$ for the indicated growth conditions [29]. The scale of the step densities increases downward and the data for successively higher temperatures are shifted for ease of comparison. The broken lines indicate the points where monolayer increments of the material were deposited. The simulations were performed on $232 \times 232$ lattices with steps running diagonally across the lattice with the parameters $E_{\mathrm{S}}=1.54 \mathrm{eV}, E_{\mathrm{N}}=0.23 \mathrm{eV}, E_{\mathrm{B}}=$ $0.175 \mathrm{eV}$ and $L=7$.

leads to a high coordination of most of the adatoms in clusters on the uppermost layer. This reduces the influence of fast processes such as free adatoms migrating to coordinated sites and the elimination of sites with low coordination. Thus, both step-edge barriers and the incorporation process are important factors for correctly reproducing the recovery curves.

\section{Surface evolution in the multilayer regime}

The inclusion of a step-edge barrier into our growth model was shown in the preceding section to have a striking effect on the comparisons with the RHEED data of Ref. [29]. In this section, we will examine the effect of this barrier on the large-scale morphology of a singular surface over longer growth periods. For a model with only deposition and site-to-site hopping, the long-time behavior of the surface is a self-organized critical state whose roughness is characterized by exponents analogous to those used for equilibrium phase transitions [34-38]. A step-edge barrier produces an altogether different type of behavior. 
Johnson et al. [11] have used the atomic force microscope to take "snapshots" of quenched $\mathrm{GaAs}(001)$ surfaces. These scans showed that the growth morphology on an initially atomically smooth surface consists of large mounds, typically $8 \mathrm{~nm}$ in height and with cross-sections of $0.5 \mu \mathrm{m} \times 1.5 \mu \mathrm{m}$ (elongated along [110]) [39]. The slope of these mounds remains approximately constant during growth. However, a vicinal surface with a misorientation above a certain critical value (i.e., with short enough terraces) shows only a small amount of statistical roughening with no evidence of mound formation. These observations suggest that growth by step flow is "stable" because a vicinal surface remains vicinal, but that growth on singular surfaces or on vicinal surfaces away from step flow is "unstable" because the mounds produce a length scale (the terrace length between successive layers of the mounds) that is enhanced during growth. The two cases are distinguished by a critical value of the misorientation which depends on the flux and the surface temperature.

To compare the morphologies reported in Ref. [11] (see also [39]), we performed large-scale simulations using the latter model of Sec. 3 . We chose the temperature and the flux to yield step flow conditions for a surface misoriented by $2^{\circ}$ (cf. Fig. 3). The evolution of the surface morphology is shown in Fig. 4. Multilayer

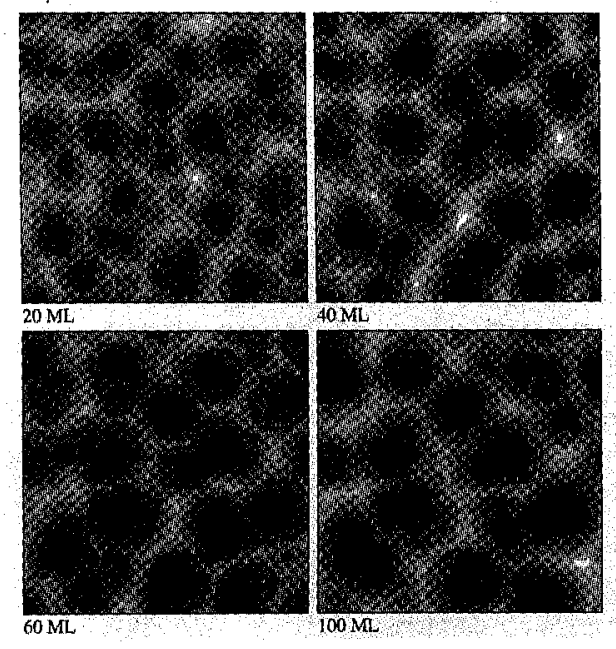

Fig. 4. Surface morphologies for the indicated deposition times produced by the model used in Sec. 3 to produce the data in Fig. 1. The shading increases with increasing height. The temperature of the substrate is $580^{\circ} \mathrm{C}$ and the growth rate is $0.20 \mathrm{ML} / \mathrm{s}$, which corresponds to conditions for step flow growth on a substrate misoriented by $2^{\circ}$ toward the [010] direction, i.e., an average terrace width of 29 lattice units. The parameters are the same as those used for the simulations in Fig. 3.

features are discernible at the earliest time shown (20 ML). As more material is deposited (40 ML), these features evolve such that the average terrace lengths fluctuate around a constant value. Further deposition (60 ML and 100 ML) causes incipient coalescence and enlargement of the features and a regularization of terrace 
lengths. These trends are in excellent qualitative agreement with those reported in Ref. [11].

The effect of including the step-edge barrier in the model can be seen by comparing the morphologies produced by simulations with and without this barrier. This is shown in Fig. 5. The morphology of the surface without the step-edge barrier is seen to be smoother than the simulation with the barrier in the sense that fewer layers are exposed. This is to be expected since when the barrier to downward hops at a step edge means that more atoms will occupy different layers than if this barrier is removed.
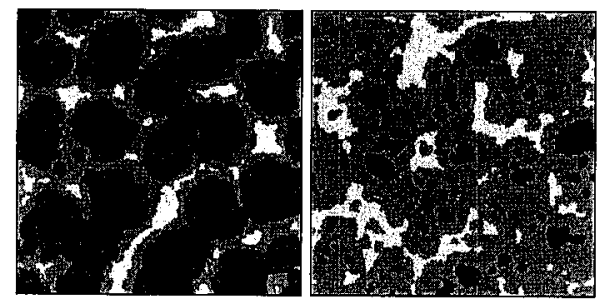

Fig. 5. Surface morphologies after the deposition of $20 \mathrm{ML}$ produced by model of epitaxial without a step-edge barrier that inhibits interlayer hopping to the lower terrace at a step edge (left panel) and with such a barrier included. The growth conditions are the same as those used to produce the morphologies in Fig. 4.

\section{Summary and conclusions}

We have addressed several issues pertaining to the evolution of the surface morphology during epitaxial growth. By making comparisons directly with experiment we were first able to optimize the parameters that enter the model and to identify the pertinent processes that characterize adatom migration. In the multilayer regime, the surface morphology showed structures similar to those seen in large area atomic force microscopy scans on quenched $\mathrm{GaAs}(001)$ surfaces. These examples illustrate how direct comparison can be used to identify not only the important kinetic processes during growth, but to determine the implications of these processes on the way the surface grows as well.

\section{References}

[1] A.Y. Cho, Thin Solid Films 100, 291 (1983).

[2] B.A. Joyce, Rep. Prog. Phys. 48, 1595 (1985).

[3] M.A. Herman, H. Sitter, Molecular Beam Epitaxy: Fundamentals and Current Status, Springer-Verlag, Berlin 1989.

[4] T. Shitara, Ph.D. thesis, University of London, 1992.

[5] Reflection High-Energy Electron Diffraction and Reflection Electron Imaging of Surfaces, Eds. P.K. Larsen, P.J. Dobson, Plenum, New York 1988.

[6] Kinetics of Ordering and Growth at Surfaces, Ed. M.G. Lagally, Plenum, New York 1990. 
[7] B. Voigtländer, A. Zinner, Appl. Phys. Lett. 63, 3055 (1993).

[8] A. Madhukar, S.V. Ghaisas, CRC Crit. Rev. Solid State Mater. Sci. 14, 1 (1988).

[9] D.D. Vvedensky, S. Clarke, K.J. Hugill, A.K. Myers-Beaghton, M.R. Wilby, in: Kinetics of Ordering and Growth at Surfaces, Ed. M.G. Lagally, Plenum, New York 1990, p. 297.

[10] H. Metiu, Y.-T. Lu, Z. Zhang, Science 255, 1088 (1992).

[11] M.D. Johnson, C. Orme, A.W. Hunt, D. Graff, J. Sudijono, L.M. Sander, B.G. Orr, Phys. Rev. Lett. 72, 116 (1994).

[12] J.D. Weeks, G.H. Gilmer, Adv. Chem. Phys. 40, 157 (1979).

[13] Z. Zhang, H. Metiu, J. Chem. Phys. 93, 2087 (1990).

[14] T. Ala-Nissila, S.C. Ying, Phys. Rev. B 42, 10264 (1990).

[15] S. Clarke, D.D. Vvedensky, Phys. Rev. Lett. 58, 2235 (1987).

[16] S.A. Barnett, A. Rockett, Surf. Sci. 198, 133 (1988).

[17] H. Yan, Phys. Rev. Lett. 68, 3048 (1992).

[18] D.A. Kessler, H. Levine, L.M. Sander, Phys. Rev. Lett. 69, 100 (1992).

[19] A. Madhukar, Surf. Sci. 132, 344 (1983).

[20] P. Smilauer, M.R. Wilby, D.D. Vvedensky, Phys. Rev. B 47, 4119 (1993).

[21] L.-M. Peng, M.J. Whelan, Proc. R. Soc. Lond. A 435, 257 (1991).

[22] S. Clarke, M.R. Wilby, D.D. Vvedensky, T. Kawamura, T. Sakamoto, Appl. Phys. Lett. 54, 2417 (1989).

[23] T. Yokotsuka, M.R. Wilby, D.D. Vvedensky, T. Kawamura, K. Fukutani, S. Ino, Appl. Phys. Lett. 62, 1673 (1993).

[24] G. Erlich, F.G. Hudda, J. Chem. Phys. 44, 1039 (1966).

[25] S.C. Wang, G. Erlich, Phys. Rev. Lett. 70, 41 (1993).

[26] R. Kunkel, B. Poelsma, L.K. Verheij, G. Comsa, Phys. Rev. Lett. 65, 733 (1990).

[27] P. Chen, J.Y. Kim, A. Madhukar, N.M. Cho, J. Vac. Sci. Technol. B 4, 890 (1986).

[28] S. Clarke, D.D. Vvedensky, M.R. Wilby, Surf. Sci. 255, 91 (1991).

[29] T. Shitara, D.D. Vvedensky, M.R. Wilby, J. Zhang, J.H. Neave, B.A. Joyce, Phys. Rev. B 46, 6815, 6825 (1992).

[30] D.D. Vvedensky, S. Clarke, Surf. Sci. 225, 373 (1990).

[31] P. Smilauer, D.D. Vvedensky, Phys. Rev. B 48, 17603 (1993).

[32] H.T.W. Zandvliet, H.B. Elswijk, D. Dijkkamp, E.J. van Loenen, J. Dieleman, J. Appl. Phys. 70, 2614 (1991).

[33] T. Shitara, J. Zhang, J.H. Neave, B.A. Joyce, Appl. Phys. Lett. 71, 4299 (1992).

[34] Z.-W. Lai, S. Das Sarma, Phys. Rev. Lett. 66, 2348 (1991).

[35] J. Villain, J. Phys. I 1, 19 (1991).

[36] S. Das Sarma, J. Vac. Sci. Technol. 10, 1695 (1992).

[37] M.R. Wilby, D.D. Vvedensky, A. Zangwill, Phys. Rev. B 46, 12896 (1992); (Errata) 47, 16068 (1993).

[38] D.D. Vvedensky, A. Zangwill, C.N. Luse, M.R. Wilby, Phys. Rev. E 48, 852 (1993).

[39] D.D. Vvedensky, Phys. World 7, 30 (1994). 